\title{
POTENCIAL EVOCADO SOMATOSSENSITIVO E MOTOR NA ESPONDILOSE CERVICAL
}

\author{
Daniela Oliveira de Andrade
}

\begin{abstract}
RESUMO - Foram estudados 54 pacientes com diagnóstico de espondilose cervical (EC) com ou sem sinais clínicos de mielopatia espondilótica. Realizou-se análise re trospectiva de todos os pacientes port ado res de EC investigados por ressonância magnética (RNM) cervical, potenciais evocados somatossensitivos (PESS) e motor (PEM) de membros superiores e inferiores. A RNM foi considerada padrão-ouro e os pacientes foram divididos em três grupos. Grupo 1 (RNM sem compressão medular), Grupo 2 (apenas indentação medular) e grupo 3 (compressão medular associada a alteração do sinal medular). A sensibilidade do PESS de quatro membros foi $61,9 \%$, similar à encontrada quando realizado o PESS apenas de membros inferiores. A sensibilidade do PEM de quatro membros foi $71,4 \%$, em membros superiores isoladamente foi $66,7 \%$ e em membros inferiores $52,4 \%$, mostrando a importância da realização deste método nos quatro membros quando suspeita-se de mielopatia espondilótica cervical. Os resultados encontrados pelo estudo do nervo tibial no PESS e do músculo abdutor do dedo mínimo no PEM mostrou maior percentual de achados alterados que os resultados encontrados pelo estudo do nervo mediano no PESS e do músculo extensor curto dos dedos no PEM nos três grupos, sugerindo que existe um comprometimento inicial deles.
\end{abstract}

PALAVRAS-CHAVE: potencial evocado somatossensitivo, potencial evocado motor, espondilose cervical, mielopatia espondilótica.

\section{Somatosensory and motor evoked potentials in patients with cervical spondylosis}

\begin{abstract}
This study investigated 54 patients with cervical spondylosis (CS) with or without symptoms caused by cervical spondylotic myelopathy. Cervical MRI, somatosensory evoked potentials (SEPS) and motor evoked potentials (MEP) from upper and lower limbs of all of these patients were examined retrospectively. We re MRI findings the gold standard and the patients were classified in three groups. Group 1 (absence of spinal cord compression ); Group 2 ( presence of spinal cord indentation); Group 3 (spinal cord compression with alteration of intraspinal sign). The sensitivity of SEP of four limbs was $61.9 \%$, the same one as the SEP of lower limbs. The sensitivity of MEP of four limbs was $71.4 \%$, of the upper limbs alone was $66.7 \%$ and of the lower limbs alone $52.4 \%$, thus showing the importance of using this method in all four limbs when cervical spondylotic myelopathy is suspected. The results shown by the study of the tibial nerve in SEP and of the abductor digiti minimi muscle in MEP were more frequently abnormals than the results shown by the study of the median nerve and extensor digitorum brevis muscle in the three groups, suggesting that exists a onset compromising of them.
\end{abstract}

KEY WORDS: motor evoked potentials, somatosensory evoked potentials, cervical spondylosis, spondylotic myelopathy.

A espondilose cervical (EC) é considerada a principal causa de mielopatia cervical após a idade de $40 \operatorname{anos}^{1-3}$ Ela também é responsável por grande $p$ a rte das radiculopatias cervicais, tendo mesmo o comprometimento radicular maior prevalência que a mielopatia espondilótica cervical. Os sintomas mais comuns dentre os pacientes com mielopatia espondilótica cervical são dormência nas mãos e dificuldade de marcha, e nos casos mais graves podem cursar com hiperreflexia global, alterações vesicais e tetraparesia. Estudos preliminares de pacientes com mielopatia espondilótica evidenciaram maior sensibilidade do potencial evocado somatossensitivo (PESS) obtido através da estimulação do neno fibular, $85,7 \%^{4}$ e $100 \%{ }^{5}$, em comparação aos potenciais obtidos da estimulação do nervo mediano, $68,5 \%{ }^{4}$ e $70 \%{ }^{5}$. Alguns trabalhos mostraram que o PESS obtido através da estimulação do nervo fibular na mielopatia espondilótica tem sensibilidade similar a do exame clínico ${ }^{4,5}$.

Tsiptsios et al. ${ }^{4}$ encontraram $77 \%$ dos PESS alterados ao ser estimulado o nervo ulnar e $68,5 \%$ ao ser estimulado o nervo mediano. Conforme o trabalho de Yiannikas et al. ${ }^{5}$ as respostas obtidas ao

Rede Sarah de Hospitais da Reabilitação: Médica Neurofisiologista Clínica do Hospital Sarah, Salvador BA, Brasil.

Recebido 27 Setembro 2004, recebido na forma final 22 Março 2005. Aceito 10 Maio 2005. 
estimular o nervo ulnar têm como desvantagem menor amplitude que as do nervo mediano. $O$ estudo da condução motora central por estimulação magnética cortical, na mielopatia espondilótica cervical, mostrou-se alterado em $84 \%^{6}$ e $100 \%{ }^{7}$ dos pacientes, através do registro com captação em membros superiores. Tavy et al. ${ }^{8}$ realizaram o único trabalho de potencial evocado motor (PEM) na mielopatia espondilótica com captação nos quat ro membros, e encontraram $96 \%$ dos PEM alterados. Os achados deste trabalho, segundo análise do autor, mostram a importância da realização deste exame com captação também em membros inferiores, e sugerem que os achados no músculo tibial anterior (TA) estão mais freqüentemente alterados que no músculo abdutor do dedo mínimo (ADM).

Tavy el al. ${ }^{9}$ realizaram estudo comparativo entre o PESS e o PEM dos quatro membros em 25 pacientes portadores de EC, com indentação ou compressão medular em estudos de neuroimagem, mas sem sinais de mielopatia ao exame clínico. Este estudo encontrou alteração apenas em $8 \%$ dos PEM e $4 \%$ do PESS, não sendo estatisticamente significante em relação ao grupo controle. Há trabalhos que evidenciaram PESS $^{4}$ e PEM $^{10}$ alterados em pacientes que não apresentam sinais ou sintomas de mielopatia, sugerindo que não apenas a compre ssão localizada mas a isquemia intermitente e persistente contribuem na fisiopatologia da mielopatia. Baseados nesses achados analisamos pacientes port a d o resde EC com e sem sinais clínicos de mielopatia buscando evidenciar a importância desses métodos, PESS e PEM, para o diagnóstico precoce de sofrimento medular, como também a contribuição isolada da análise em membros superiores e inferiores na sensibilidade de cada técnica.

\section{MÉTODO}

Foi realizada análise tipo corte transversal de 54 pacientes port a d o res de EC. Esses pacientes foram selecionados dentre 88 pacientes, que corre spondem à população total com esta patologia atendida neste hospital de janeiro de 2002 a dezembro de 2003. Foram excluídos pacientes que apresentassem neuropatia periférica, história de lesão medular por outra causa ou outro comprometimento do sistema nervoso central (SNC), como cirurgias prévias da coluna ou encéfalo. Os pacientes foram classificados como portadores de mielopatia pela presença de déficit motor associado a sinais de liberação piramidal. Todos os dados foram colhidos dos prontuários eletrônicos.

Todos os pacientes analisados realizaram ressonância magnética (RNM) da coluna cervical, PESS e PEM neste hospital. Eles foram divididos em três g rupos considerando a presença ou não de compressão medular à RNM: Grupo 1 pacientes sem compressão medular; Grupo 2 pacientes com indentação da medula; Grupo 3 com compressão e alteração do sinal medular.

Os pacientes foram investigados com PESS e PEM no mesmo dia e com intervalo máximo de um ano da realização da RNM.

Os exames de PESS foram realizados com os pacientes em posição supina usando aparelho Keypoint (Dantec, Skovlunde, Denmark). O PESS foi re alizado por estímulo elétrico percutâneo no nervo mediano bilateral nos membros superiores (MMSS) e tibial posterior bilateral nos membros inferiores (MMII) na intensidade suficiente para produzir leve contração muscular involuntária deflagrados por estimulador de superfície com duração de $0,2 \mathrm{~ms}$ e freqüência de $1,0 \mathrm{~Hz}$ no total de 500 estímulos em cada segmento.

PESS do nervo mediano foi obtido após captação com eletrodos de superfície (disco $5 \mathrm{~mm}$ ) na região supra-clavicular (ponto de Erb), processo espinhoso cervical C7 e no escalpe em C3' e C4' (sistema internacional 10-20) em montagem referencial Erb contralateral e $\mathrm{Fz}$ respectivamente. $\mathrm{O}$ PESS do nervo tibial bilateral foi obtido após captação na região lombar L1-L2, cervical C7 e no escalpe em Cz. Eletrodos de referência colocados na crista ilíaca e Fz. Impedância mantida abaixo de $5 \Omega \mathrm{Ohm}$. Filtros de $50-1000 \mathrm{~Hz}$. Os componentes examinados foram latências absolutas e interpicos de N9, N13 e N19 nos MMSS e latências absolutas e interpicos de PL, N30 e N/P37 nos MMII.

As vias motoneuronais foram estudadas utilizando o PEM Magpro (Dantec, Skovlunde, Denmark). Durante o procedimento o paciente foi colocado em posição supina e relaxado. Pulsos magnéticos liberados através de bobina circular MC125 de diâmetro externo de 120 mm, gerando um campo máximo de 2,2 Tesla no centro da bobina. A intensidade do estímulo foi $80 \%$ da máxima de saída para captação em MMSS e $100 \%$ da máxima de saída para captação em MMII, emitindo pulsos bifásicos e amplificados com filtros de $20 \mathrm{~Hz}$ e $3 \mathrm{kHz}$, freqüência máxima de $1,0 \mathrm{~Hz}$ e duração de $0,2 \mathrm{~ms}$. Foram realizados estímulos corticais com bobina posicionada no vértex para captação nos MMSS e C3'e C4'contralaterais à captação nos MMII (sistema internacional 10-20). Durante os estímulos os pacientes realizaram leve contração do músculo estudado com intuito de facilitar a resposta. O potencial de ação muscular composto (PAMC) foi capta- 
do por eletrodos de superfície posicionados sobre o ventre muscular (ponto motor) dos músculos extensor curto dos dedos (EDB) nos MMII e abdutor do dedo mínimo (ADM) nos MMSS. Quatro estímulos subseqüentes e seus potenciais foram registrados de cada sítio de estimulação. O tempo de condução motora central (TCMC) foi calculado da subtração da menor latência cortical, da latência periférica (TCMC=LC-LP). A latência periférica é $M+F-$ $1 / 2^{11}$, onde $M$ corresponde a latência da onda $M$ e $F$ a latência da onda $F$.

$O$ PESS e o PEM foram considerados alterados quando o tempo de condução central em um dos quatromembros estivesse prolongado ou o potencial cortical ausente. Para análise do PEM levou-se em consideração a razão das amplitudes dos potenciais corticais e a onda M (PEM/M). A análise considerou como valores normais a média $\pm 3 \mathrm{DP}$ dos achados dos PESS ${ }^{12}$ e PEM ${ }^{13}$ descritos na literatura e similar aos valores encontrados em estudo de normatização de grupo controle de 103 pacientes realizados neste hospital ${ }^{14}$.

\section{RESULTADOS}

A idade dos pacientes variou de 34 a 77 anos (média 52,2 anos); 25 (46,3\%) eram homens e 29 $(53,7 \%)$ mulheres.

Do total de pacientes avaliados $10(18,5 \%)$ foram classificados no Grupo 1, $23(42,6 \%)$ no Grupo 2 e $21(38,9 \%)$ no Grupo 3.

No Grupo 1 e 2 apenas $4(12,1 \%)$ pacientes apresentaram sinais clínicos de mielopatia, e no Grupo $3,9(42,8 \%)$ pacientes.

Dos 54 pacientes estudados 24 apresentaram PESS alterados, $11(33,4 \%)$ no Grupo 1 e 2 e 13 $(61,9 \%)$ no Grupo 3. O PEM evidenciou 32 exames alterados, $17(81 \%)$ no Grupo 1 e 2 e $15(71,4 \%)$ no Grupo 3 (Tabela 1).

Considerou-se para análise da sensibilidade e especificidade dos métodos PESS e PEM os pacientes do Grupo 1 e 2 como pacientes de um mesmo grupo sem compressão medular significativa. O Grupo 3 composto de pacientes com compressão medular à RNM.

A sensibilidade do PESS em relação aos achados da RNM cervical foi $61,9 \%(38,7-81,0)$ I.C.95\% e especificidade $66,7 \%(48,1-81,4)$ I.C. $95 \%$, e a sensibilidade do PEM foi $71,4 \%(47,7 \%-87,7 \%)$ I.C.95\% e especificidade $48,5 \%(31,2 \%-66,1 \%)$ I.C.95\%. A sensibilidade do PESS em MMSS isoladamente foi $19 \%$ e em MMII 61,9\%. A sensibilidade do PEM em MMSS foi $66,7 \%$ e em MMII 52,4\%.

Dentre os pacientes que apresentaram PESS alterados (Tabela 2) nos Grupos 1 e 2, nenhum apresentou alteração do exame nos MMSS e todos apresentaram alteração apenas nos MMII. No Grupo 3, nenhum paciente apresentou alteração isolada nos MMSS, $69 \%$ apresentaram alteração exclusiva dos MMII, e $31 \%$ em 3 ou 4 membros.

Dentre os pacientes que apresentaram PEM alterados (Tabela 2), no Grupo 1, 66,7\% apresentaram alteração restrita aos MMSS, 16,6\% apenas nos MMII e 16,7\% apresentaram alteração em 3 ou 4 membros. No grupo 2, 54,5\% dos pacientes apresentaram alteração apenas em MMSS, nenhum paciente apresentou alteração restrita aos MMII, e 45,5\% dos pacientes apresentaram alteração em 3 ou 4 membros. No Grupo 3, 26,7\% apresentaram alteração restrita aos MMSS, 6,6\% nos MMII, e um maior percentual correspondendo a $67 \%$ em 3 a 4 membros.

Tabela 1. Achados do potencial evocado somatossensitivo e motor nos quatro membros quanto a presença ou não de compressão medular à RNM cervical.

\begin{tabular}{lccccccccccccccccc}
\hline Membros & \multicolumn{3}{c}{ Grupo 1 } & \multicolumn{4}{c}{ Grupo 2} & \multicolumn{4}{c}{ Grupo 3 } & \multicolumn{3}{c}{ Total } \\
& \multicolumn{1}{c}{ PESS } & PEM & PESS & PEM & PESS & PEM & PESS & PEM \\
& $\mathrm{n}$ & $\%$ & $\mathrm{n}$ & $\%$ & $\mathrm{n}$ & $\%$ & $\mathrm{n}$ & $\%$ & $\mathrm{n}$ & $\%$ & $\mathrm{n}$ & $\%$ & $\mathrm{n}$ & $\%$ & $\mathrm{n}$ & $\%$ \\
\hline 1MI & 1 & 1,85 & 1 & 1,85 & 4 & 7,4 & 0 & 0 & 4 & 7,4 & 1 & 1,85 & 9 & 16,7 & 2 & 3,7 \\
1MS & 0 & 0 & 1 & 1,85 & 0 & 0 & 4 & 7,4 & 0 & 0 & 3 & 5,6 & 0 & 0 & 8 & 14,85 \\
1MS e 1MI & 0 & 0 & 0 & 0 & 0 & 0 & 0 & 0 & 0 & 0 & 0 & 0 & 0 & 0 & 0 & 0 \\
2MMII & 2 & 3,7 & 0 & 0 & 4 & 7,4 & 0 & 0 & 5 & 9,3 & 0 & 0 & 11 & 20,4 & 0 & 0 \\
2MMSS & 0 & 0 & 3 & 5,55 & 0 & 0 & 2 & 3,7 & 0 & 0 & 1 & 1,85 & 0 & 0 & 6 & 11,1 \\
2MMSS e 1MI & 0 & 0 & 0 & 0 & 0 & 0 & 1 & 1,85 & 1 & 1,8 & 1 & 1,85 & 1 & 1,8 & 2 & 3,7 \\
1MS e 2MMII & 0 & 0 & 0 & 0 & 0 & 0 & 1 & 1,85 & 3 & 5,6 & 1 & 1,85 & 3 & 5,6 & 2 & 3,7 \\
2MMSS e 2MMII & 0 & 0 & 1 & 1,85 & 0 & 0 & 3 & 5,6 & 0 & 0 & 8 & 14,8 & 0 & 0 & 12 & 22,25 \\
Normal & 7 & 12,95 & 4 & 7,4 & 15 & 27,8 & 12 & 22,2 & 8 & 14,8 & 6 & 11,1 & 30 & 55,5 & 22 & 40,7 \\
Total & 10 & 18,5 & 10 & 18,5 & 23 & 42,6 & 23 & 42,6 & 21 & 38,9 & 21 & 38,9 & 54 & 100 & 54 & 100 \\
\hline
\end{tabular}

MI, membro inferior; MS, membro superior; MMII, membros inferiores; MMSS, membros superiores. PESS, Potencial evocado somatossensitivo; PEM, Potencial evocado motor. 
Tabela 2. Percentual de pacientes quanto ao número de membros alterados no Potencial evocado somatossensitivo e potencial evocado motor, nos três diferentes grupos.

\begin{tabular}{lcccccc}
\hline Membros alterados & \multicolumn{2}{c}{ Grupo 1} & \multicolumn{2}{c}{ Grupo 2} & \multicolumn{2}{c}{ Grupo 3 } \\
& PESS & PEM & PESS & PEM & PESS & PEM \\
\hline $1 \mathrm{MI}$ & $33,4 \%$ & $16,6 \%$ & $50 \%$ & $0 \%$ & $30 \%$ & $6,6 \%$ \\
$1 \mathrm{MS}$ & $0 \%$ & $16,7 \%$ & $0 \%$ & $36,3 \%$ & $0 \%$ & $20 \%$ \\
$2 \mathrm{MMII}$ & $66,6 \%$ & $0 \%$ & $50 \%$ & $0 \%$ & $38,5 \%$ & $0 \%$ \\
$2 \mathrm{MMSS}$ & $0 \%$ & $50 \%$ & $0 \%$ & $18,2 \%$ & $0 \%$ & $6,7 \%$ \\
3 ou 4MM & $0 \%$ & $16,7 \%$ & $0 \%$ & $45,5 \%$ & $31 \%$ & $66,7 \%$ \\
Total & $100 \%$ & $100 \%$ & $100 \%$ & $100 \%$ & $100 \%$ & $100 \%$ \\
\hline
\end{tabular}

MI, membro inferior; MS, membro superior; MMII, membros inferiores; MMSS, membros superiores; 3 ou 4 MM, 3 ou 4 membros; PESS, Potencial evocado sematossensitivo; PEM, Potencial evocado motor.

\section{DISCUSSÃO}

Alguns trabalhos avaliaram a sensibilidade do PESS $^{5,6}$ e outros do PEM $^{7,8}$ na mielopatia espondilótica cervical e considerando os valores descritos, o PEM tem evidenciado sensibilidade superior ao PESS. Este trabalho comparou os dois exames, PESS e PEM, estudando-os nos quatro membros no mesmo grupo de indivíduos. Nos três grupos estudados o PEM mostrou maior sensibilidade que o PESS em concordância com os achados da literatura.

Os pacientes incluídos neste estudo apresentaram um baixo percentual de sinais clínicos de mielopatia. Mesmo no Grupo 3 apenas 10/21 (45, $5 \%$ ) dos pacientes apresentavam sinais clínicos de comprometimento medular, motivo que contribuiu para a baixa sensibilidade encontrada em relação aos valores descritos em outros estudos ${ }^{4-8}$.

Este trabalho evidenciou percentual de alterados nos PEM e PESS superior ao encontrado no exame clínico, o que sugere a possibilidade dessas técnicas de diagnosticar disfunção medular em pacientes sem marcadores clínicos de mielopatia.

A sensibilidade do PESS em MMII foi maior que nos MMSS, sendo este achado similar ao descrito na literatura 5 . A sensibilidade do PESS em MMII é similar à sensibilidade do exame nos quatro membros na EC. Todos os PESS anormais no Grupo $1 \mathrm{e}$ 2 apresentavam alterações nos MMII (fascículo grácil). Apesar de não ter sido realizado estudo longitudinal dos pacientes, o predomínio do comprometimento dos membros inferiores nos três grupos de gravidade diferentes e a ausência de comprometimento isolado do membro superior, sugere haver lesão inicial comprometendo o fascículo grácil e comprometimento do cuneiforme apenas nos casos mais avançados.

Este estudo evidenciou que o PEM tem maior sensibilidade quando realizado nos quatro mem$b$ ros. O PEM evidenciou maior sensibilidade à captação no músculo ADM em relação ao músculo
EDB. Encontrou-se maior percentagem de pacientes com início das alterações nos MMSS no Grupo $1(66,7 \%)$, Grupo $2(54,5 \%)$ e Grupo $3(26,7 \%)$, que pacientes com alterações isoladas nos MMII, G rupo 1 (16,6\%), Grupo 2 ( $0 \%$ ) e Grupo 3 (6,6\%). Esses achados sugerem um comprometimento preferencial das fibras corticoespinhais referentes aos membros superiores na espondilose cervical.

\section{REFERÊNCIAS}

1. Irvine DH, Foster JB, Newell DJ, Klukvin BN. Prevalence of cervical spondylosis in a general practice. Lancet 1965;14:1089-1092.

2. Wilkinson M. The morbid anatomy of cervical spondylosis and myelopathy. Brain 1978;83:589-617.

3. Crandall PH, Gregorius F K. Long term follow-up of surgical treatment of cervical spondylotic myelopathy. Spine 1977;2:139-146.

4. Tsiptsios I, Fotiou F, Sitzoglou K, Fountoulakis KN. Neurophysiological investigation of cervical spondylosis. Electromyogr Clin Neurophysiol 2001;41:305-313.

5. Yiannikas C, Shahani BT, Young RR. Short-latency somatosensory-evoked potentials from radial, median, ulnar, and peroneal nerve stimulation in the assessment of cervical spondylosis. Arch Neurol 1986; 43:1264-1271.

6. Maertens de Noordhout A, Remacle JM, Pepin JL, Born JD, Delwaide PJ. Magnetic stimulation of the motor cortex in cervical spondylosis. Neurology 1991;41:75-80.

7. Di Lazzaro V, Restuccia D, Colosimo C, Tonali P. The contribution of magnetic stimulation of the motor cortex to the diagnosis of cervical spondylotic myelopathy: correlation of central motor conduction to distal and proximal upper limb muscles with clinical and MRI findings. Electroenceph Clin Neurophysiol 1992;85:311-320.

8. Tavy DLJ, Louis Wagner G, Keunen RWM, Rolf Wattendorff A, Hekster REM, Frassen H. Transcranial magnetic stimulation in patients with cervical spondylotic myelopathy: clinical and radiological correlations. Muscle Nerve 1994; 17: 235-241.

9. Tavy DLJ, Frassen H, Keunen RWM, Wattendorff AR, Hekster REM, Van Huffelen AC. Motor and somatosensory evoked potentials is asymptomatic spondylotic cord compression. Muscle Nerve 1999;22:628-634.

10. Di Lazzaro V, Oliviero A, Profice P, et al. The diagnostic value of motor evoked potentials. Clin Neurophysiol 1999;110:1297-1307.

11. Rossini PM, Barker AT, Berardelli A, et al. Non-invasive electrical and magnetic stimulation of the brain, spinal cord and roots: basic principles and procedures for routine clinical application. Electroenceph Clin Neurophysiol 1994;91:79-82.

12. Chiappa KH. Short-latency somatosensory evoked potentials: methodology. In Chiappa KH (ed). Evoked potentials in clinical medicine. New York: Raven Press, 1989:307-370.

13. Dvorák J, Herdmann J, Vohánka S. Motor evoked potentials by means of magnetic stimulation in disorders of the spine. Methods Clin Neurophysiol 1992;3:45-64.

14. Fonte Boa PMO. Potencial evocado motor por estimulação magnética transcraniana e radicular: estudo normativo para os músculos abdutor digiti minimi e extensor digitorum brevis. Dissertação (Mestrado), Faculdade de Medicina de Ribeirão Preto, Universidade de São Paulo. Ribeirão Preto, 1997. 